\title{
Variety of orthomodular posets
}

\author{
Ivan Chajda and Miroslav Kolarik
}




\title{
VARIETY OF ORTHOMODULAR POSETS
}

\author{
IVAN CHAJDA AND MIROSLAV KOLAŘÍK
}

Received 24 September, 2013

\begin{abstract}
Orthomodular posets play an important role in the so-called logical structure of a physical system as formerly pointed out by numerous authors. In particular, they play an essential role in the logic of quantum mechanics. To avoid usual problems with partial algebras, we define the so-called orthomodular directoid as an everywhere defined algebra and we show that every orthomodular poset can be converted into an orthomodular directoid and vice versa. Since orthomodular directoids are defined equationally, they form a variety having nice congruence properties.
\end{abstract}

2010 Mathematics Subject Classification: 06C15; 06A06

Keywords: orthomodular poset, orthomodular lattice, orthomodular directoid, variety of algebras

\section{INTRODUCTION}

By a logical structure of a physical system (see $[1,2,14]$ or [13]) is meant a couple $(L ; F)$, where $L$ is a nonvoid set and $F$ is a set of functions from $L$ into the interval $[0,1]$ of real numbers satisfying the following axioms:

(I) If $p, q \in L$ and $f(p)=f(q)$ for every $f \in F$ then $p=q$.

(II) There exists an element $u \in L$ such that $f(u)=1$ for each $f \in F$.

(III) For each $p \in L$, there exists an element $p^{\prime} \in L$ such that $f(p)+f\left(p^{\prime}\right)=1$ for every $f \in F$.

Let $\leq$ be the relation defined on $L$ by

$$
p \leq q \text { if and only if } f(p) \leq f(q) \text { for every } f \in F .
$$

Then $\leq$ is a partial order on $L$ with the least element $u^{\prime}$ and the greatest element $u$. We say that $p, q \in L$ are orthogonal if $p \leq q^{\prime}$ (which is equivalent to $q \leq p^{\prime}$, see [1] for details).

We add one more axiom:

Support of the research of the first author by the Project CZ.1.07/2.3.00/20.0051 "Algebraic Methods in Quantum Logics" and of the second author by the Project CZ.1.07/2.3.00/20.0060 "International Center for Information and Uncertainty" is gratefully acknowledged. 
(IV) For every orthogonal elements $p, q \in L$ there exists supremum $s=\sup (p, q)$ and $f(s)=f(p)+f(q)$ for each $f \in F$.

It is well-known that the system $(L ; \leq,, 0,1)$ is an orthomodular poset, the so-called associated poset with the logical structure $(L ; F)$, see e.g. [1]. Hence, orthomodular posets serve as an axiomatic description of physical systems, see e.g. [5,7]. If $\sup (p, q)$ exists for each couple $p, q$ of elements of $L$, then $\left(L ; \leq,{ }^{\prime}, 0,1\right)$ becomes an orthomodular lattice. Hence, the theory of orthomodular posets includes the theory of orthomodular lattices and, simultaneously, serves as an axiomatization of the logic of physical systems. In particular, it axiomatizes the logic of quantum mechanics, see $[2,7,10,14]$ and $[13]$.

Due to the above mentioned properties, orthomodular posets were and are studied by numerous authors for several decades see e.g. [5,7,8,12,13]. However, up to now, orthomodular posets were treated as partial algebras where the binary operation of supremum is ensured only for orthogonal or comparable elements. In this paper, we try another approach, namely to introduce a certain everywhere defined algebra which can be assigned to every orthomodular poset in the way that the underlying poset coincides with the original one but its axioms can be expressed as identities. Hence, the class of these so-called orthomodular directoids forms a variety of algebras having nice algebraic properties. Moreover, every orthomodular poset can be recovered by means of this assigned algebra despite the fact that the assignment need not be done in a unique way.

\section{ORTHOMODULAR DIRECTOIDS}

Recall by [9] (see also [4]) that a groupoid $(A ;+)$ is called a commutative directoid if it satisfies the following axioms:

$$
\begin{aligned}
& x+x=x \\
& x+y=y+x \\
& x+((x+y)+z)=(x+y)+z .
\end{aligned}
$$

In what follows, we enrich the commutative directoid by a unary operation (orthocomplementation) and by two constants to get an algebra for our study. Since we need to ask for two more properties connected with orthomodular posets (namely the orthomodular law and the existence of suprema for orthogonal elements), we add two more axioms which caused that some other axioms for orthomodular directoids can follow from the remaining ones. Hence, we can define:

Definition 1. By an orthomodular directoid is called an algebra $\mathscr{D}=\left(D ;+,{ }^{\prime}, 0,1\right)$ of type $(2,1,0,0)$ satisfying the following axioms:

(D1) $x+y=y+x$

(D2) $x+((x+y)+z)=(x+y)+z$

(D3) $x+0=x$

(D4) $x+x^{\prime}=1$ 
(D5) $\left(\left((x+z)+(y+z)^{\prime}\right)^{\prime}+(y+z)^{\prime}\right)+z^{\prime}=z^{\prime}$

(D6) $x+\left(x+(x+y)^{\prime}\right)^{\prime}=x+y$.

Theorem 1. The axioms (D1)-(D6) are independent.

Proof. (a) Define $0^{\prime}=1, a^{\prime}=1,1^{\prime}=0,0+a=1, a+a=a, 1+a=1$ and $x+0=x, y+1=1$ for each $x, y \in\{0, a, 1\}$. Then $\left(\{0, a, 1\} ;+,^{\prime}, 0,1\right)$ satisfies (D2)-(D6), but not (D1) because $a+0=a \neq 1=0+a$.

(b) Define' and + by the following tables

\begin{tabular}{l|llllllllllll|}
$x$ & 0 & $a$ & $b$ & $c$ & $d$ & $e$ & $f$ & $g$ & $h$ & $i$ & $j$ & 1 \\
\hline$x^{\prime}$ & 1 & $b$ & $a$ & $d$ & $c$ & $f$ & $e$ & $h$ & $g$ & $j$ & $i$ & 0
\end{tabular}

\begin{tabular}{|c|cccccccccccc|}
\hline+ & 0 & $a$ & $b$ & $c$ & $d$ & $e$ & $f$ & $g$ & $h$ & $i$ & $j$ & 1 \\
\hline 0 & 0 & $a$ & $b$ & $c$ & $d$ & $e$ & $f$ & $g$ & $h$ & $i$ & $j$ & 1 \\
$a$ & $a$ & $a$ & 1 & $g$ & $d$ & 1 & 1 & $g$ & $d$ & 1 & 1 & 1 \\
$b$ & $b$ & 1 & $b$ & $b$ & 1 & 1 & 1 & 1 & $b$ & 1 & 1 & 1 \\
$c$ & $c$ & $g$ & $b$ & $c$ & 1 & 1 & 1 & $g$ & $b$ & 1 & 1 & 1 \\
$d$ & $d$ & $d$ & 1 & 1 & $d$ & 1 & 1 & 1 & $d$ & 1 & 1 & 1 \\
$e$ & $e$ & 1 & 1 & 1 & 1 & $e$ & 1 & $i$ & $h$ & $i$ & $h$ & 1 \\
$f$ & $f$ & 1 & 1 & 1 & 1 & 1 & $f$ & $f$ & 1 & 1 & $f$ & 1 \\
$g$ & $g$ & $g$ & 1 & $g$ & 1 & $i$ & $f$ & $g$ & 1 & $i$ & $f$ & 1 \\
$h$ & $h$ & $d$ & $b$ & $b$ & $d$ & $h$ & 1 & 1 & $h$ & 1 & $h$ & 1 \\
$i$ & $i$ & 1 & 1 & 1 & 1 & $i$ & 1 & $i$ & 1 & $i$ & 1 & 1 \\
$j$ & $j$ & 1 & 1 & 1 & 1 & $h$ & $f$ & $f$ & $h$ & 1 & $j$ & 1 \\
1 & 1 & 1 & 1 & 1 & 1 & 1 & 1 & 1 & 1 & 1 & 1 & 1 \\
\hline
\end{tabular}

Then $\left(\{0, a, b, c, d, e, f, g, h, i, j, 1\} ;+,^{\prime}, 0,1\right)$ satisfies (D1), (D3)-(D6), but not (D2) because

$$
a+((a+c)+e)=a+i=1 \neq i=(a+c)+e .
$$

(c) Define $0^{\prime}=1,1^{\prime}=0$ and the constant operation $x+y=1$ for every $x, y \in$ $\{0,1\}$. Then $\left(\{0,1\} ;+,,^{\prime}, 0,1\right)$ satisfies (D1), (D2) and (D4)-(D6), but not (D3) because $0+0=1 \neq 0$.

(d) Suppose now that + is commutative and $x+0=x=x+x$ for each $x \in$ $\{0, a, b, 1\}$. Further let $a+b=a+1=b+1=a, 0^{\prime}=a, a^{\prime}=0, b^{\prime}=1$ and $1^{\prime}=b$. Then $\left(\{0, a, b, 1\} ;+,{ }^{\prime}, 0,1\right)$ satisfies (D1)-(D3), (D5) and (D6), but not (D4) because $a+a^{\prime}=a \neq 1$.

(e) Define + and ' by the following tables 


\begin{tabular}{|c|cccccc|}
\hline+ & 0 & $a$ & $b$ & $c$ & $d$ & 1 \\
\hline 0 & 0 & $a$ & $b$ & $c$ & $d$ & 1 \\
$a$ & $a$ & $a$ & 1 & $c$ & $d$ & 1 \\
$b$ & $b$ & 1 & $b$ & $c$ & $d$ & 1 \\
$c$ & $c$ & $c$ & $c$ & $c$ & 1 & 1 \\
$d$ & $d$ & $d$ & $d$ & 1 & $d$ & 1 \\
1 & 1 & 1 & 1 & 1 & 1 & 1 \\
\hline
\end{tabular}

Then $\left(\{0, a, b, c, d, 1\} ;+{ }^{\prime}, 0,1\right)$ satisfies (D1)-(D4) and (D6), but not (D5) because

$$
\begin{gathered}
\left(\left((1+a)+(c+a)^{\prime}\right)^{\prime}+(c+a)^{\prime}\right)+a^{\prime} \\
=\left(\left(1+c^{\prime}\right)^{\prime}+c^{\prime}\right)+a^{\prime}=(0+d)+b=d \neq b=a^{\prime} .
\end{gathered}
$$

(f) Finally, define $0^{\prime}=1^{\prime}=1$ and $0+0=0,0+1=1+0=1+1=1$. Then $\left(\{0,1\} ;+,{ }^{\prime}, 0,1\right)$ satisfies (D1)-(D5), but not (D6) because $0+\left(0+(0+0)^{\prime}\right)^{\prime}=0+$ $1=1 \neq 0=0+0$.

We can derive several more useful identities satisfied by orthomodular directoids.

Lemma 1. Every orthomodular directoid satisfies the following:
(a) $x^{\prime \prime}=x$
(b) $x+1=1$
(c) $x+x=x$
(d) $0^{\prime}=1$ and $1^{\prime}=0$
(e) $\left(x^{\prime}+y\right)^{\prime}+x=x$.

Proof. (a) Putting $x=0$ and $y=x$ in (D6) we obtain $0+\left(0+(0+x)^{\prime}\right)^{\prime}=0+x$. Applying (D1) and (D3) four times we get $x^{\prime \prime}=x$.

(b) Put $y=x^{\prime}$ and $z=0$ in (D2). We get $x+\left(\left(x+x^{\prime}\right)+0\right)=\left(x+x^{\prime}\right)+0$ whence $x+1=1$ using (D3) and (D4).

(c) By (D2) where $y=z=0$ we have $x+((x+0)+0)=(x+0)+0$. Applying (D3) four times we conclude $x+x=x$.

(d) Putting $x=0$ in (D4) we obtain $1=0+0^{\prime}=0^{\prime}$ by (D1) and (D3). Hence, using (a), $0=1^{\prime}$.

(e) Substitute $y$ instead of $x$ and $x^{\prime}$ instead of $z$ in (D5). Using (a) we get

$$
\left(\left(\left(y+x^{\prime}\right)+\left(y+x^{\prime}\right)^{\prime}\right)^{\prime}+\left(y+x^{\prime}\right)^{\prime}\right)+x=x
$$

whence $\left(1^{\prime}+\left(y+x^{\prime}\right)^{\prime}\right)+x=x$ by (D4). Applying (d), (D1) and (D3) we conclude $\left(x^{\prime}+y\right)^{\prime}+x=x$.

Lemma 2. Let $\mathfrak{D}=\left(D ;+,,^{\prime}, 0,1\right)$ be an orthomodular directoid. Define a binary relation $\leq$ on $D$ as follows

$$
x \leq y \quad \text { if and only if } \quad x+y=y .
$$

Then $\leq i$ a partial order on $D$ such that: 
(a) $0 \leq x \leq 1$ for each $x \in D$

(b) $x \leq x+y, y \leq x+y$

(c) $x \leq y$ implies $y^{\prime} \leq x^{\prime}$

(d) if $x+y=0$ then $x=y=0$

(e) if $x+(x+y)^{\prime}=1$ then $y \leq x$.

Proof. By (c) of Lemma 1 we see that $\leq$ is reflexive. Assume $x \leq y$ and $y \leq x$. Then $x+y=y$ and $y+x=x$. Due to (D1) we infer $x=y$, i.e. $\leq$ is antisymmetrical. If $x \leq y$ and $y \leq z$ then $x+y=y, y+z=z$ and, applying (D2), we have

$$
x+z=x+(y+z)=x+((x+y)+z)=(x+y)+z=y+z=z
$$

showing $x \leq z$, transitivity of $\leq$. Hence, $\leq$ is a partial order on $D$. By (D3) and (D1), $0 \leq x$ and, by (b) of Lemma $1, x \leq 1$ for each $x \in D$.

If we put $z=0$ in (D2), we obtain $x+(x+y)=x+y$ whence $x \leq x+y$. Analogously $y \leq y+x=x+y$.

Further, assume $x \leq y$, i.e. $x+y=y$. Then, by (e) and (a) of Lemma 1, we infer $(x+y)^{\prime}+x^{\prime}=x^{\prime}$ and hence $y^{\prime}+x^{\prime}=x^{\prime}$, proving $y^{\prime} \leq x^{\prime}$.

The assertion (d) follows from (b).

Finally, assume $x+(x+y)^{\prime}=1$. By (D6) we infer $x+y=x+\left(x+(x+y)^{\prime}\right)^{\prime}=$ $x+1^{\prime}=x+0=x$ thus $y \leq x$.

The partial order defined by $(*)$ will be referred to as the induced order of $\mathscr{D}=$ $\left(D ;+,{ }^{\prime}, 0,1\right)$.

Now, we recall the concept of orthomodular poset (from [1]).

Definition 2. By an orthomodular poset is meant a structure $\mathcal{P}=\left(P ; \leq,^{\prime}, 0,1\right)$, where $\leq$ is a partial order on $P, 0 \leq x \leq 1$ for each $x \in P, x^{\prime \prime}=x, x^{\prime}$ is a complement of $x$ and $x \leq y$ implies $y^{\prime} \leq x^{\prime}$, and satisfying the following two conditions:

(i) if $x \leq y^{\prime}$ then the set $\{x, y\}$ has the supremum $x \vee y$ in $(P ; \leq)$

(ii) if $x \leq y$ then $x \vee\left(x \vee y^{\prime}\right)^{\prime}=y$.

Remark 1. (a) Since $x \leq x$ for each $x \in P, x \vee x^{\prime}$ exists and $x \vee x^{\prime}=1$.

(b) Since $x \leq y$ implies $y^{\prime} \leq x^{\prime}$, the existence of $x \vee y$ yields the existence of $x^{\prime} \wedge y^{\prime}=(x \vee y)^{\prime}$, the infimum of $x^{\prime}, y^{\prime}$, by De Morgan laws. In particular, $x \vee x^{\prime}=1$ and $x^{\prime \prime}=x, 1^{\prime}=0$ get immediately $x^{\prime} \wedge x=0$ and hence $x^{\prime}$ is a complement of $x$.

(c) If $x \leq y$ then, by (i), $x \vee y^{\prime}$ exists. Since $x \leq x \vee y^{\prime}$, also $x \vee\left(x \vee y^{\prime}\right)^{\prime}$ exists thus (ii) is correctly defined. By using De Morgan laws, (ii) can be read as follows:

$$
x \leq y \quad \Rightarrow \quad x \vee\left(x^{\prime} \wedge y\right)=y
$$

which is the orthomodular law. Hence, if $x \vee y$ exists for each $x, y \in P$ then $\mathcal{P}=\left(P ; \leq,{ }^{\prime}, 0,1\right)$ is an orthomodular lattice (see $\left.[1,11]\right)$.

By (i), if $x, y$ are orthogonal then $x \vee y$ exists. Of course, $x \vee y$ exists also for comparable elements since $x \leq y$ gets $x \vee y=y$. 
If $\mathcal{P}=\left(P ; \leq,{ }^{\prime}, 0,1\right)$ is an orthomodular lattice then the orthomodular law (OML) can be expressed in the form of identity as follows:

$$
x \vee\left(x^{\prime} \wedge(x \vee y)\right)=x \vee y .
$$

(d) If $x \leq a$ and $y \leq a^{\prime}$ for some $a \in P$ then $x \vee y$ exists. Namely, $y \leq a^{\prime}$ yields $a \leq y^{\prime}$ thus $x \leq a \leq y^{\prime}$ implies that $x, y$ are orthogonal and, by (i) of Definition $2, x \vee y$ exists in $(P ; \leq)$.

Example 1. See [1]. Let $M$ be a finite set with an even number of elements. Let $P$ be the set of all subsets of $M$ which have even number of elements ordered by inclusion and let $A^{\prime}=M \backslash A$, the set-theoretical complementation. Then $\mathcal{P}=(P ; \subseteq$ $,, \varnothing, M)$ is an orthomodular poset. If $|M| \geq 6$ then $\mathcal{P}$ is not a lattice.

Now, we are going to show that every orthomodular directoid is an orthomodular poset. For this, let us firstly prove the following lemma.

Lemma 3. Let $\mathscr{D}=\left(D ;+,{ }^{\prime}, 0,1\right)$ be an orthomodular directoid and $\leq$ its induced order.

$$
\text { If } x \leq y^{\prime} \text { then } x+y=x \vee y .
$$

Proof. In fact, we are going to prove that (2.1) is equivalent to the axiom (D5). At first we show that (2.1) is equivalent with the following condition:

$$
\left(x \leq y^{\prime} \text { and } x, y \leq z\right) \text { implies } x+y \leq z .
$$

$(2.1) \Rightarrow(2.2)$ : Assume $x \leq y^{\prime}$ and let $x, y \leq z$. By (b) of Lemma 2 we have $x, y \leq$ $x+y$ thus (2.1) yields (2.2) immediately.

(2.2) $\Rightarrow$ (2.1): Let $x \leq y^{\prime}$ and $x, y \leq z$. By (2.2), $x+y$ is the least common upper bound of $x, y$ thus $x+y=x \vee y$.

And now, the axiom (D5) is clearly equivalent to the condition

$$
\left((x+z)+(y+z)^{\prime}\right)^{\prime}+(y+z)^{\prime} \leq z^{\prime} .
$$

It remains to show that (2.3) is equivalent to (2.2).

(2.2) $\Rightarrow$ (2.3): Assume that (2.2) holds and put $a^{\prime}=(x+z)+(y+z)^{\prime}, b^{\prime}=y+z$. Then for $a=\left((x+z)+(y+z)^{\prime}\right)^{\prime}$ and $b=(y+z)^{\prime}$ we have $(x+z)+(y+z)^{\prime} \geq$ $(y+z)^{\prime}$ thus $a=\left((x+z)+(y+z)^{\prime}\right)^{\prime} \leq y+z=b^{\prime}$. Further, $y+z \geq z$ thus $b=(y+$ $z)^{\prime} \leq z^{\prime}$ and $(x+z)+(y+z)^{\prime} \geq x+z \geq z$, whence $a=\left((x+z)+(y+z)^{\prime}\right)^{\prime} \leq z^{\prime}$. Applying (2.2) for $a, b$ and $z^{\prime}$ we conclude $\left((x+z)+(y+z)^{\prime}\right)^{\prime}+(y+z)^{\prime} \leq z^{\prime}$ which is (2.3).

(2.3) $\Rightarrow$ (2.2): Assume that (2.3) holds and let $x \leq y^{\prime}, x, y \leq z$. Then $y \leq x^{\prime}$, i.e. $x^{\prime}+y=x^{\prime}$ and $z^{\prime} \leq x^{\prime}, y^{\prime}$, i.e. $x^{\prime}+z^{\prime}=x^{\prime}, y^{\prime}+z^{\prime}=y^{\prime}$. By (2.3) we compute

$$
x+y=x^{\prime \prime}+y=\left(x^{\prime}+y\right)^{\prime}+y=\left(\left(x^{\prime}+z^{\prime}\right)+\left(y^{\prime}+z^{\prime}\right)^{\prime}\right)^{\prime}+\left(y^{\prime}+z^{\prime}\right)^{\prime} \leq z
$$

proving (2.2). 


\section{A REPRESENTATION OF ORTHOMODULAR POSETS}

Now, we are ready to prove our first main theorem.

Theorem 2. Let $\mathscr{D}=\left(D ;+,{ }^{\prime}, 0,1\right)$ be an orthomodular directoid and $\leq$ be its induced order. Then $\mathcal{P}(D)=\left(D ; \leq,{ }^{\prime}, 0,1\right)$ is an orthomodular poset where for orthogonal elements $x, y \in D$ we have

$$
x+y=x \vee y .
$$

Proof. By Lemma 2, $(D ; \leq, 0,1)$ is a bounded poset where the mapping $x \mapsto x^{\prime}$ is an antitone involution.

Assume $x, y$ are orthogonal, i.e. $x \leq y^{\prime}$. By Lemma 3 we have $x+y=x \vee y$.

Since $x \leq x$, the elements $x, x^{\prime}$ are orthogonal thus, by (D4), $x \vee x^{\prime}=x+x^{\prime}=1$, i.e. $x^{\prime}$ is a complement of $x$.

It remains to prove the orthomodular law. Assume $x \leq y$. Then the elements $x, y^{\prime}$ are orthogonal and hence $x+y^{\prime}=x \vee y^{\prime}$ and, similarly, $x+\left(x \vee y^{\prime}\right)^{\prime}=x \vee\left(x \vee y^{\prime}\right)^{\prime}$. Because $x+y=y$, using (D6) we obtain

$$
x \vee\left(x \vee y^{\prime}\right)^{\prime}=x+\left(x+y^{\prime}\right)^{\prime}=x+\left(x+(x+y)^{\prime}\right)^{\prime}=x+y=y
$$

which is (ii) of Definition 2.

Moreover, we are able to prove the converse.

Theorem 3. Let $\mathcal{P}=\left(P ; \leq,{ }^{\prime}, 0,1\right)$ be an orthomodular poset. Define a binary operation + on $P$ as follows:

- $x+y=x \vee y$ if $x \vee y$ exists

- $x+y=y+x$ is an arbitrary element of $U(x, y)=\{z \in P ; x, y \leq z\}$ otherwise.

Then $\mathscr{D}(P)=\left(P ;+,{ }^{\prime}, 0,1\right)$ is an orthomodular directoid.

Proof. By definition, + satisfies (D1). Since $x+y \in U(x, y)$ in each case, it is elementary to check (D2). Since $0 \leq x$, we have $x=x \vee 0=x+0$ proving (D3). Since $x \vee x^{\prime}=1$, we obtain (D4). Since $x \leq x+y$, we obtain (D6) by using of the orthomodular law. It remains to prove (D5).

Put $a=\left((x+z)+(y+z)^{\prime}\right)^{\prime}, b=(y+z)^{\prime}$. Then $a=\left((x+z)+(y+z)^{\prime}\right)^{\prime} \leq$ $y+z=b^{\prime}$, i.e. $a+b=a \vee b$. However, $(x+z)+(y+z)^{\prime} \geq x+z \geq z$ thus $a \leq z^{\prime}$. Evidently, $b=(y+z)^{\prime} \leq z^{\prime}$ and hence $a+b=a \vee b \leq z^{\prime}$. Thus $(a+b)+z^{\prime}=z^{\prime}$ which is (D5).

By Theorem 3 , to every orthomodular poset $\mathcal{P}=\left(P ; \leq,^{\prime}, 0,1\right)$ can be assigned an everywhere defined algebra which is an orthomodular directoid $\mathscr{D}(P)=$ $\left(P ;+,{ }^{\prime}, 0,1\right)$. By Theorem 2 , to the orthomodular directoid $\mathscr{D}(P)$ can be assigned an orthomodular poset $\mathscr{P}(\mathscr{D}(P))$. Since the underlying posets $(P ; \leq)$ coincide in all $\mathcal{P}, \mathscr{D}(P)$ and $\mathscr{P}(\mathscr{D}(P))$ and the complementation is also the same, we conclude that $\mathcal{P}=\mathscr{P}(\mathscr{D}(P))$. Hence, although the directoid $\mathscr{D}(P)$ need not be assigned in a 
unique way, it bears all the information on $\mathcal{P}$ because $\mathcal{P}=\mathscr{P}(\mathscr{D}(P))$ for every such a directoid.

On the contrary, if $\mathscr{D}=\left(D ;+,{ }^{\prime}, 0,1\right)$ is an orthomodular directoid, $\mathcal{P}(D)$ the assigned orthomodular poset and $\mathscr{D}(\mathcal{P}(D))$ the assigned orthomodular directoid then $\mathscr{D}$ and $\mathscr{D}(\mathscr{P}(D))$ need not be even isomorphic because the operation + in $\mathscr{D}(\mathcal{P}(D))$ can be choosen differently than that in $\mathscr{D}$.

Theorem 4. Let $\mathscr{D}=\left(D ;+,{ }^{\prime}, 0,1\right)$ be an orthomodular directoid, $\leq$ its induced order and $a \in D$. Then $\left([a, 1] ;+,{ }^{a}, a, 1\right)$ for $x^{a}=x^{\prime}+a$ is an orthomodular directoid.

Proof. Assume $a \in D$ and $x \in[a, 1]$. Then $a \leq x$ and hence $a \vee x^{\prime}$ exists and $x^{a}=x^{\prime}+a=x^{\prime} \vee a$. Since $x^{\prime} \vee a \geq a$, also $a \vee\left(a \vee x^{\prime}\right)^{\prime}$ exists and $x^{a a}=\left(x^{\prime} \vee\right.$ $a)^{\prime} \vee a=a$ by the orthomodular law. Moreover, $x \leq y$ for $x, y \in[a, 1]$ yields $y^{a}=$ $y^{\prime} \vee a \leq x^{\prime} \vee a=x^{a}$ and $x^{a}=x^{\prime} \vee a \geq a$, i.e. $x \mapsto x^{a}$ is an antitone involution on the interval $[a, 1]$. Further, $x^{a}=x^{\prime} \vee a \geq x^{\prime}$ thus $\left(x^{a}\right)^{\prime} \vee x^{\prime}$ exists and, by De Morgan laws, $\left(x^{a}\right)^{\prime} \vee x^{\prime}=\left(x^{a} \wedge x\right)^{\prime}=\left(\left(x^{\prime} \vee a\right) \wedge x\right)^{\prime}=a^{\prime}$ by the orthomodular law. Therefore $x^{a} \wedge x=a$ and $x^{a} \vee x=x^{a} \vee x^{a a}=\left(x \wedge x^{a}\right)^{a}=a^{a}=1$, thus $x^{a}$ is an orthocomplement of $x$ in the interval $[a, 1]$. It is plain to check the orthomodular law in the poset $\left([a, 1] ; \leq,{ }^{a}, a, 1\right)$ and hence the induced algebra $\left([a, 1] ;+{ }^{a}, a, 1\right)$ is an orthomodular directoid.

\section{THE VARIETY OF ORTHOMODULAR DIRECTOIDS}

By Theorems 2 and 3, orthomodular posets can be represented by everywhere defined algebras, i.e. by orthomodular directoids. However, by Definition 1, these directoids are determined by the identities (D1)-(D6) and hence the class $\mathcal{K}$ of orthomodular directoids forms a variety of algebras. In what follows, we present several important properties of the variety $\mathcal{K}$.

Recall that an algebra $\mathcal{A}$ is congruence distributive if its congruence lattice $\operatorname{Con} \mathcal{A}$ is distributive. A variety $\mathcal{V}$ is congruence distributive if each $\mathcal{A} \in \mathcal{V}$ has this property. By a majority term is meant a ternary term $m(x, y, z)$ such that

$$
m(x, x, y)=m(x, y, x)=m(y, x, x)=x \text {. }
$$

It follows directly by the Jónsson characterization that a variety $\mathcal{V}$ having a majority term is congruence distributive, see e.g. [3].

Theorem 5. The variety $\mathcal{K}$ of orthomodular directoids is congruence distributive.

Proof. Consider the ternary term

$$
m(x, y, z)=((x \sqcap y)+(y \sqcap z))+(x \sqcap z),
$$

where $a \sqcap b=\left(a^{\prime}+b^{\prime}\right)^{\prime}$. It is immediately clear that $a \sqcap a=a, a \sqcap b=b \sqcap a$, $a \sqcap b \leq a$ and $a \sqcap b \leq b$, thus $(a \sqcap b)+a=a$ and $(a \sqcap b)+b=b$. Hence

$$
m(x, x, y)=((x \sqcap x)+(x \sqcap y))+(x \sqcap y)=(x+(x \sqcap y))+(x \sqcap y)
$$




$$
\begin{aligned}
& =x+(x \sqcap y)=x \\
m(x, y, x) & =((x \sqcap y)+(y \sqcap x))+(x \sqcap x)=(x \sqcap y)+x=x \\
m(x, y, y) & =((x \sqcap y)+(y \sqcap y))+(x \sqcap y)=((x \sqcap y)+y)+(x \sqcap y) \\
& =y+(x \sqcap y)=y
\end{aligned}
$$

proving that $m$ is a majority term of $\mathcal{K}$.

Let us recall (see e.g. [3]) that an algebra $\mathcal{A}$ is congruence regular if every congruence on $\mathcal{A}$ is determined by every its class, i.e. if for any $\Theta, \Phi \in$ Con $\mathcal{A}$ and each $a \in A$, if $[a]_{\Theta}=[a]_{\Phi}$ then $\Theta=\Phi$. A variety $\mathcal{V}$ is congruence regular if each $\mathcal{A} \in \mathcal{V}$ has this property. The following result was proved by B. Csákány, see [6].

Proposition 1. A variety $\mathcal{V}$ is congruence regular if and only if there exists $n \geq 1$ and ternary terms $t_{1}, \ldots, t_{n}$ such that

$$
\left(t_{1}(x, y, z)=z \text { and } \ldots \text { and } t_{n}(x, y, z)=z\right) \text { if and only if } x=y .
$$

Using this, we prove the following result.

Theorem 6. The variety of orthomodular directoids is congruence regular.

Proof. At first, consider the term

$$
x \triangle y=\left(x+(x+y)^{\prime}\right)^{\prime}+\left(y+(x+y)^{\prime}\right)^{\prime} .
$$

If $x=y$ then clearly $\left(x+(x+y)^{\prime}\right)^{\prime}=\left(x+x^{\prime}\right)^{\prime}=1^{\prime}=0$ and hence $x \Delta x=0$. Conversely, assume $x \Delta y=0$. By (d) of Lemma 2, $\left(x+(x+y)^{\prime}\right)^{\prime}=0$ and $(y+$ $\left.(x+y)^{\prime}\right)^{\prime}=0$, i.e. $x+(x+y)^{\prime}=1$ and $y+(x+y)^{\prime}=1$. By (e) of Lemma 2, $y \leq x$ and $x \leq y$ giving $x=y$.

Now, take $n=2$ and consider the terms $t_{1}(x, y, z)=(x \triangle y)+z, t_{2}(x, y, z)=$ $\left((x \Delta y)+z^{\prime}\right)^{\prime}$. If $x=y$ then

$$
t_{1}(x, x, z)=(x \triangle x)+z=0+z=z
$$

and

$$
t_{2}(x, x, z)=\left((x \triangle x)+z^{\prime}\right)^{\prime}=\left(0+z^{\prime}\right)^{\prime}=z^{\prime \prime}=z .
$$

Conversely, assume $t_{1}(x, y, z)=t_{2}(x, y, z)=z$. Then $(x \Delta y)+z=z$, i.e. $x \Delta y \leq$ $z$ and $\left((x \Delta y)+z^{\prime}\right)^{\prime}=z$, that is, $(x \Delta y)+z^{\prime}=z^{\prime}$. Thus we get $x \Delta y \leq z^{\prime}$, whence $z \leq(x \Delta y)^{\prime}$. Together we have $x \Delta y \leq z \leq(x \Delta y)^{\prime}$ which is possible if and only if $x \Delta y=0$. As shown above, it gets $x=y$. Hence $t_{1}, t_{2}$ are the terms of the Proposition proving congruence regularity.

Recall that a variety $\mathcal{V}$ is permutable if $\Theta \circ \Phi=\Phi \circ \Theta$ for every $\mathcal{A} \in \mathcal{V}$ and each $\Theta, \Phi \in \operatorname{Con} \mathcal{A}$. As proved by A.I. Mal'cev (see e.g. [3]), a variety $\mathcal{V}$ is permutable if and only if there exists a ternary term $p(x, y, z)$ such that $p(x, x, z)=z$ and $p(x, z, z)=x$.

Theorem 7. The variety of orthomodular directoids is permutable. 
Proof. In Theorem 4, the involution $x^{a}$ for $x \in[a, 1]$ has been introduced. Since $y \leq x+y$, we have $x+y \in[y, 1]$ and hence $(x+y)^{y}=(x+y)^{\prime}+y$ is defined. As shown by Theorem 4, we have $(x+y)^{y y}=x+y$ and $(x+y)^{y} \geq y$ whence $(x+y)^{y}+y=(x+y)^{y}$. Define

$$
p(x, y, z)=\left((z+y)^{y}+x\right)^{x} \sqcap\left((x+y)^{y}+z\right)^{z},
$$

where again $a \sqcap b=\left(a^{\prime}+b^{\prime}\right)^{\prime}$. Then

$$
\begin{gathered}
p(x, x, z)=\left((z+x)^{x}+x\right)^{x} \sqcap\left((x+x)^{x}+z\right)^{z} \\
=(z+x)^{x x} \sqcap(1+z)^{z}=(z+x) \sqcap z=z
\end{gathered}
$$

and, analogously,

$$
p(x, z, z)=\left((z+z)^{z}+x\right)^{x} \sqcap\left((x+z)^{z}+z\right)^{z}=x \sqcap(x+z)=x .
$$

Thus $p$ is a Mal'cev term, and hence the variety of orthomodular directoids is permutable.

\section{REFERENCES}

[1] L. Beran, Orthomodular lattices. Algebraic approach, 1st ed., ser. Mathematics and Its Applications (East European Series). Prague: Academia, 1985, vol. XVIII.

[2] G. Birkhoff and J. von Neumann, "The logic of quantum mechanics," Ann. of Math., vol. 37, no. 2, pp. 823-843, 1936.

[3] I. Chajda, G. Eigenthaler, and H. Länger, Congruence Classes in Universal Algebra, 1st ed., ser. Research and Exposition in Mathematics. Wien: Heldermann Verlag, 2003, vol. XXVI.

[4] I. Chajda and H. Länger, Directoids. An algebraic approach to ordered sets, 1st ed., ser. Research and Exposition in Mathematics. Wien: Heldermann Verlag, 2011, vol. XXXII.

[5] I. Chajda and H. Länger, "Spaces of abstract events," Int. J. Theor. Phys., vol. 52, no. 6, pp. 1818-1824, 2013.

[6] B. Csákány, "Characterizations of regular varieties," Acta Sci. Math., vol. 31, no. 1, pp. 187-189, 1970.

[7] K. Engesser, D. Gabbay, and D. Lehmann, Handbook of quantum logic and quantum structures, 1st ed. Amsterdam: Elsevier Science, 2009.

[8] B. Jeffcott, "The center of an orthologic," J. Symbolic Logic, vol. 37, no. 4, pp. 641-645, 1973.

[9] J. Ježek and R. Quackenbush, "Directoids: Algebraic models of up-directed sets," Algebra Univers., vol. 27, no. 1, pp. 49-69, 1990.

[10] G. Kalmbach, "Orthomodular logic," Z. Math. Logik Grundlagen Math., vol. 20, no. 1, pp. 395406, 1974

[11] G. Kalmbach, Orthomodular lattices, 1st ed., ser. London Mathematical Society Monographs. London: Academic Press, 1983, vol. VIII.

[12] M. Matoušek and P. Pták, "Orthocomplemented posets with a symmetric difference," Order, vol. 26, no. 1, pp. 1-21, 2009.

[13] N. Megill and M. Pavičić, "Orthomodular lattices and a quantum algebra," Int. J. Theor. Phys., vol. 40, no. 8, pp. 1387-1410, 2001

[14] C. Randall and D. Foulis, "An approach to empirical logic," Am. Math. Mon., vol. 77, no. 4, pp. 363-374, 1970. 
Authors' addresses

Ivan Chajda

Palacký University Olomouc, Faculty of Science, Department of Algebra and Geometry, 17. listopadu 12, 77146 Olomouc, Czech Republic

E-mail address: ivan.chajda@upol.cz

Miroslav Kolařík

Palacký University Olomouc, Faculty of Science, Department of Computer Science, 17. listopadu 12, 77146 Olomouc, Czech Republic

E-mail address: miroslav.kolarik@upol.cz 